\title{
Highway Performance Evaluation Index in Semiarid Climate Region Based on Fuzzy Mathematics
}

\author{
Sanqiang Yang, ${ }^{1,2}$ Meng Guo $\mathbb{D D}^{3}{ }^{3}$ Xinlei Liu, ${ }^{1}$ Pidong Wang, ${ }^{1}$ Qian Li, ${ }^{1}$ and Haiqing Liu ${ }^{3}$ \\ ${ }^{1}$ Baoding City Road and Bridge Engineering Assembly Technology Key Laboratory, College of Civil Engineering and Architecture, \\ Hebei University, Baoding, Hebei 071002, China \\ ${ }^{2}$ Hebei Institute of Transportation Planning and Design, Shi Jiazhuang, Hebei 050011, China \\ ${ }^{3}$ The Key Laboratory of Urban Security and Disaster Engineering of Ministry of Education, Beijing University of Technology, \\ Beijing 100124, China
}

Correspondence should be addressed to Meng Guo; mguo@ustb.edu.cn

Received 27 February 2019; Revised 27 April 2019; Accepted 14 May 2019; Published 17 June 2019

Academic Editor: Mohammad A. Hariri-Ardebili

Copyright (c) 2019 Sanqiang Yang et al. This is an open access article distributed under the Creative Commons Attribution License, which permits unrestricted use, distribution, and reproduction in any medium, provided the original work is properly cited.

\begin{abstract}
Accurate evaluation and analysis of expressway pavement performance is a prerequisite for determining the pavement design scheme and maintenance scheme. Due to the fuzziness and randomness of many factors affecting the pavement performance, this paper relies on the reconstruction and expansion project of Xinglin section of the Taihang mountain expressway, a method of highway pavement performance evaluation based on fuzzy mathematics is proposed. The results show the following: (1) the study uses the factor domain, the comment level domain, the fuzzy relationship matrix, the evaluation factor full vector, and the fuzzy comprehensive evaluation result vector five-step method. The method can be effectively combined with the multi-index comprehensive detection index used in the specification. (2) Based on the multi-index comprehensive test and evaluation adopted in the specification, the performance grade of the old road surface was quantitatively evaluated by the iterative calculation of fuzzy mathematics that broke through the evaluation mode which was based on the traditional detection methods. The research results provide innovative theoretical methods for the accurate evaluation and analysis of highway pavement performance in the semiarid climate region and also play a technical supporting role for the pavement design scheme and maintenance scheme decision-making in the semiarid climate region.
\end{abstract}

\section{Background}

By the end of 2017, the total mileage of expressways in China is close to $130,000 \mathrm{~km}$, among which the service period of 5-10 years accounts for up to $45 \%$. The performance evaluation of the old road surface and the design of the pavement maintenance scheme will determine the investment budget for the reconstruction of the old road network [1].Pavement performance evaluation is based on the collected pavement condition data to judge the degree of pavement performance to meet the requirements of use. It is helpful to understand the pavement condition and the change rule of service performance, making reasonable countermeasures, and is the basis of making corresponding maintenance and reconstruction measures [2]. Pavement performance generally remains good for 1 to 5 years. In 6 to 15 years, pavement performance is degraded due to natural environment factors, traffic loads, and temperature changes. A series of diseases such as ruts and cracks appear on the road surface. Under the comprehensive influence of various factors $[3,4]$, the asphalt pavement of expressway needs a large area of maintenance for 5 to 8 years, resulting in a large capital investment. Therefore, in order to maintain the level of highway service, delaying the decline of road performance, reducing asset losses, extending the service life of the road surface are the main tasks of highway maintenance management [5].

In recent years, many experts and scholars at home and abroad have conducted a large number of theoretical studies 
on pavement suitability evaluation and prediction. Developed countries engaged in this research earlier, and its research ideas and methods have been mostly in the establishment of the road management system and road maintenance system to achieve. Among them, a representative study has been developed by the U.S. Army Construction Engineering Research Institute of the PAVER system. The penalty method was first used to establish the pavement damage evaluation model by considering the road condition information, condition evaluation, and prediction. The method can accurately calculate and reduce overall damage caused by a variety of damage of pavement [6]. The Arizona management system used the evenness and cracking data to evaluate the pavement performance [7].The Milligan state road management system management proposed the road performance attenuation curve, the forecast road performance [8]. In 2001, Prozzi from the University Of California proposed a method to analyze and evaluate pavement performance by combining field test data with experimental data [9]. In the same year, Nunoo from Florida International University proposed to adopt the composite comprehensive evaluation algorithm to optimize the multiyear integrated pavement maintenance plan for the network pavement management and maintenance problems [10]. The SHRP, which began in 1987, considers pavement long-term performance (LTPP) research as a 10year highway research project with the goal of providing the means and assistance to "improve pavement performance and service life" [11]. In 2001, Hao from Chang'an University analyzed the necessity, research plan, and research strategy of carrying out long-term pavement performance research in his doctoral thesis "pavement performance evaluation and analysis research" [12].

In terms of pavement performance evaluation, there are generally two categories of evaluation indexes: single index and comprehensive index. The evaluation model mainly includes PSI [13] of AASHO in the United States, Canada's RCI [14], Japan's MCI, and China's PQI and RQI. This paper relies on the research carried out by the Chinese project, so the Chinese PCI and RQI models are selected.

Hebei Province is a typical semiarid climate region. At present, the total length of expressways in Hebei Province is $6531 \mathrm{~km}$. The performance evaluation of old highway pavement is especially difficult. The semiarid climate area has less precipitation, strong sunshine, and sharp temperature change. The shrinkage deformation of highway pavement in the semiarid climate area is particularly prominent due to high temperature rutting in summer, low temperature cracking in winter, annual temperature difference, and diurnal temperature difference.

In recent ten years, researchers began to apply fuzzy mathematics to highway performance evaluation index research. Zhou et al. proposed a prediction method of pavement performance based on grey prediction theory and clustering analysis method [15]. Chen established the evaluation model of highway asphalt pavement which is created on the basis of fuzzy evaluation, grey relationship, and ameliorated artificial neutral network (ANN) [16]. In highway pavement maintenance management decision- making optimization, Wen discussed highway pavement maintenance project decision-making and maintenance fund allocation [17]. Li put forward a method of expressway asphalt pavement maintenance decision-making based on the matter-element model. The model can greatly reduce the calculation amount of the optimization analysis process of each maintenance section [18].

Based on the above background, this paper relies on the reconstruction and expansion project of Xinglin section of the Taihang mountain expressway, and a method of highway pavement performance evaluation based on fuzzy mathematics is proposed.

\section{Climate Characteristics and Engineering Description of Semiarid Areas}

The semiarid climate zone refers to the zone whose mean annual precipitation is less than the evaporation [19]; the drought index AI (the ratio of annual precipitation and the annual evaporation) is in the range of 0.2 to 0.5 , the precipitation is commonly $200 \sim 400 \mathrm{~mm}$, the temperature changes sharply, and the annual sunshine amount varies greatly. The difference in sunshine is more than 30 degrees Celsius, and the highest temperature can reach 75 degrees Celsius. The areas include the central and eastern Inner Mongolia, Hebei, Yanbei in Shanxi, northern region of Shanxi, Xihaigu in the south of Ningxia, Dingxi in Gansu, Yuzhong, Yushu in Qinghai, Guoluo, Lhasa in Tibet, and other regions. Rainfall is directly related to pavement water resistance, and temperature difference is directly related to pavement high and low temperature performance. Correlation analysis based on fuzzy mathematics can solve multifactor problems better.

The Xingtai-Linqing expressway based on this study is located in the south of Hebei Province, with gentle topography and topographic forms of alluvial, diluvial, and limnetic plain. For the warm temperate semiarid monsoon climate region, weather is like in spring drought windy, summer weather hot and rainy, and winter weather cold and less snowy. The annual average temperature is $13.1^{\circ} \mathrm{C}$; the average temperature is $26.9^{\circ} \mathrm{C}$. In July, the extreme maximum temperature is $41.8^{\circ} \mathrm{C}$; the average temperature from December to January is minus 3.5 degrees Celsius, and the extreme minimum temperature is $-21.6^{\circ} \mathrm{C}$. The average annual precipitation is about $550 \mathrm{~mm}$. Through the investigation and analysis of road conditions, it is found that the sections with high temperature rutting in summer are significantly distributed, and the transverse and longitudinal cracks of the road surface are widely distributed. The types of pavement diseases are closely related to the temperature and precipitation in this area.

\section{Evaluation and Analysis of the Existing Standard Indexes}

In this study, the highway from Xingtai to Linqing in the semiarid climate region of Hebei Province was selected, and the pavement performance index (PCI) [20], road driving quality index (RQI), and rutting depth evaluation (RDI) 
were used to analyze the change rules of various pavement performance indexes from 2011 to 2016. Pavement structural strength $[21,22]$ was used to evaluate the variation rules of deflection in 2015 and 2016 (Figures 1-6).

According to Figures 1-3, from 2011 to 2016, the overall trend of the PCI index of pavement damage decreased year by year. The road surface driving quality index (RQI) is all above 90 points, indicating that the road surface is in good condition. The rutting depth index (RDI) of the road surface showed that the rutting depth index of the road surface decreased continuously with the increase of years, and the decay law of the road surface performance was obvious. It can be concluded from the analysis that the rutting depth index RDI affecting the road surface of this section is related to the quality of internal pavement material and pavement structure, as well as external climatic conditions and traffic conditions.

The bending and subsidence data of the Taihang mountain expressway from Xingtai to Linqing in the semiarid climate region of Hebei Province in 2015 and 2016 were selected for analysis, and the strength variation rules were evaluated (Figures 4-6).

According to the analysis in Figures 4-6, SSI data of the road surface from Xingtai to Linqing section of the Taihang mountain expressway in 2015 and 2016 show that, with the increase of the service period, the overall strength of the road surface structure presents a downward trend. The main factors of intensity attenuation are heavy traffic, large temperature difference, and other meteorological and environmental factors.

\section{Comprehensive Evaluation of Pavement Performance Based on Fuzzy Mathematics}

\subsection{Analysis Steps}

\subsubsection{Determine the Factor Domain of the Evaluation Object.} The evaluation fuzzy phenomenon first determines the evaluation factors, and the collection of these factors constitutes the evaluation factors [23]. The theory of domain of our country's current technical specification for the highway asphalt pavement maintenance JTG comprehensive evaluation index PQI H20-2007 is composed of four indexes, namely, the pavement damage index, roughness, rut, and antisliding performance. So the factors concerning domain $U=$ (PCI, RQI, RDI, SRI).

4.1.2. Domain the Evaluation Grade Theory. Domain the evaluation grade theory divides the evaluation objects into several levels, generally from 4 levels to 9 levels [24]. In this paper, the evaluation is divided into five grades, namely, the evaluation theory of field $V=$ (excellent, good, medium, secondary, bad $)=(A, B, C, D, E)$.

4.1.3. Establish the Fuzzy Relation Matrix. After the hierarchical fuzzy subset is constructed, the evaluated objects should be quantified from each factor one by one, that is, the membership degree of the evaluated objects to the

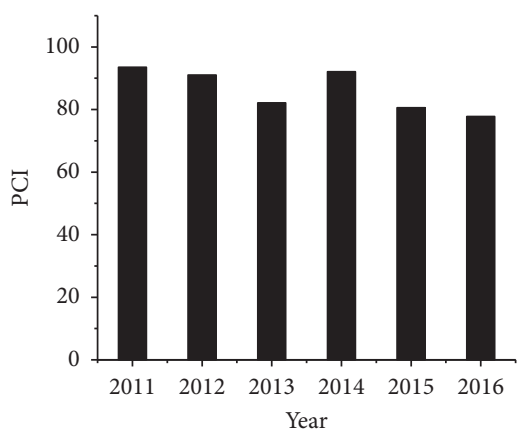

Figure 1: Continuous chart of the road condition index.

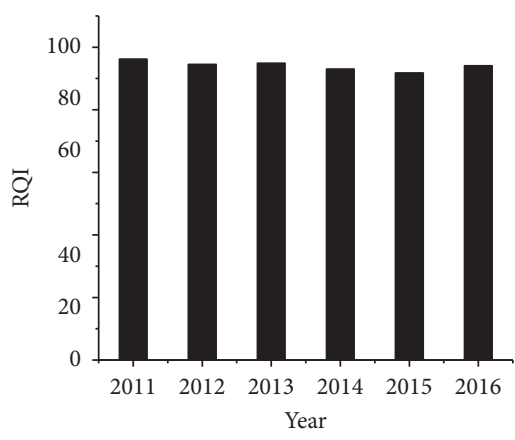

FIgURE 2: Continuous statistics of the driving quality index chart.

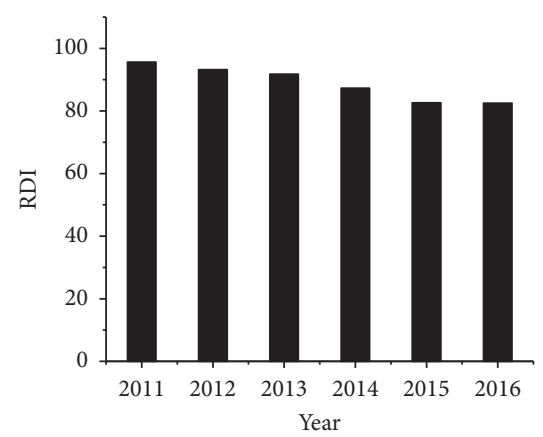

Figure 3: Continuous chart of the rutting depth index.

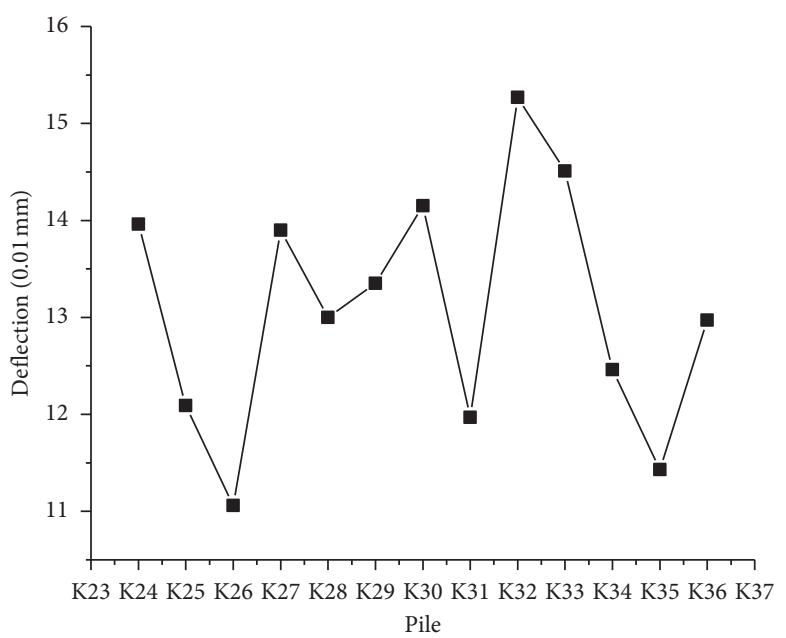

Figure 4: Trend of pavement deflection in 2015. 


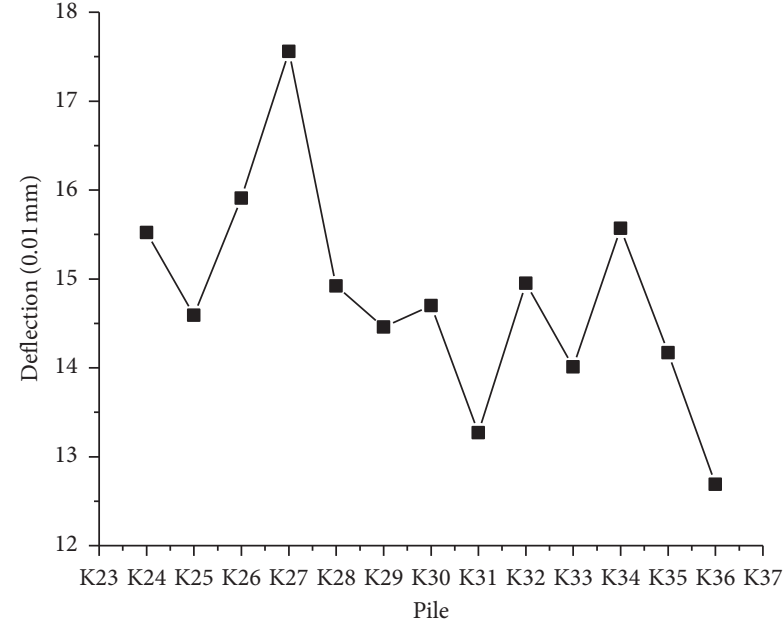

Figure 5: Trend of pavement deflection in 2016.

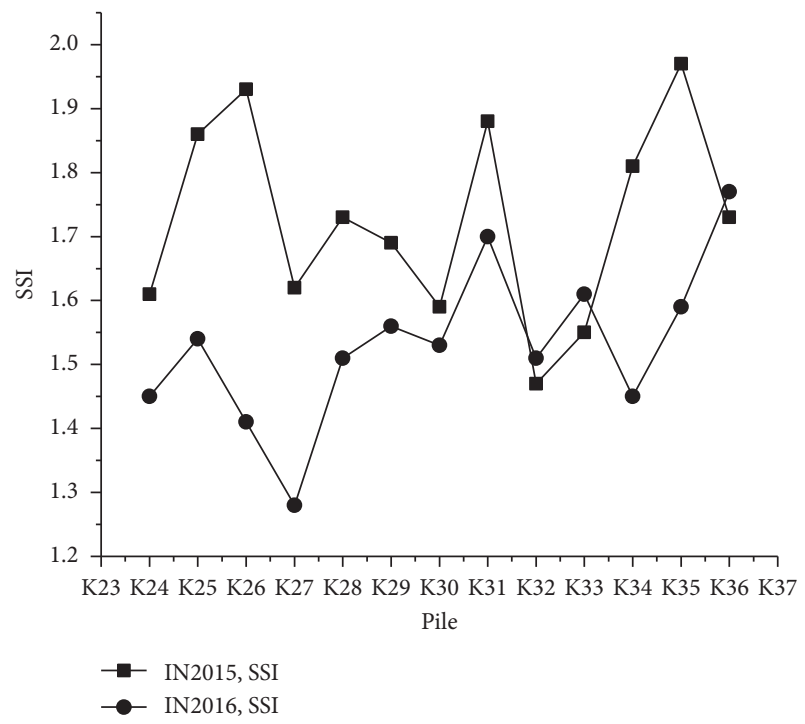

FIGURE 6: SSI comparison in 2015 and 2016.

TABLE 1: Road surface performance.

\begin{tabular}{lccccc}
\hline Indicators & Excellent & Good & Medium & Secondary & Bad \\
\hline PCI & {$[85,100)$} & {$[70,85)$} & {$[55,85)$} & {$[40,55)$} & {$[0,40)$} \\
RQI & {$[85,100)$} & {$[70,85)$} & {$[55,85)$} & {$[40,55)$} & {$[0,40)$} \\
RDI & {$[85,100)$} & {$[70,85)$} & {$[55,85)$} & {$[40,55)$} & {$[0,40)$} \\
SRI & {$[85,100)$} & {$[70,85)$} & {$[55,85)$} & {$[40,55)$} & {$[0,40)$} \\
\hline
\end{tabular}

hierarchical fuzzy subset from the perspective of a single factor [25]. The attribute measure function of each evaluation index is shown in the following table.

As can be seen from Table 1, the attribute measurement functions of the four evaluation indicators are the same. Then, the membership functions of PCI, RQI, RDI, and SRI are as follows:

$$
\begin{aligned}
& r_{\mathrm{E}}= \begin{cases}1, & 85<r \leq 100, \\
\frac{r-70}{85-70}, & 70<r \leq 85, \\
0, & 0<r \leq 75,\end{cases} \\
& r_{\mathrm{G}}= \begin{cases}\frac{100-r}{100-85}, & 85<r \leq 100, \\
1, & 70<r \leq 85, \\
\frac{r-55}{70-55}, & 55<r \leq 70, \\
0, & 0<r \leq 55,\end{cases}
\end{aligned}
$$$$
r_{\mathrm{M}}= \begin{cases}0, & 85<r \leq 100, \\ \frac{85-r}{85-70}, & 70<r \leq 85 \\ 1, & 55<r \leq 70 \\ \frac{r-40}{55-40}, & 40<r \leq 55 \\ 0, & 0<r \leq 40\end{cases}
$$$$
r_{\mathrm{S}}= \begin{cases}0, & 70<r \leq 100, \\ \frac{70-r}{70-55}, & 55<r \leq 70 \\ 1, & 40<r \leq 55 \\ \frac{r-25}{40-25}, & 25<r \leq 40 \\ 0, & 0<r \leq 25\end{cases}
$$$$
r_{\mathrm{B}}= \begin{cases}0, & 55<r \leq 100, \\ \frac{55-r}{55-40}, & 40<r \leq 55 \\ 1, & 25<r \leq 40 \\ \frac{r-10}{25-10}, & 10<r \leq 25 \\ 0, & 0<r \leq 10\end{cases}
$$ 
4.1.4. Determine the Total Vector of Evaluation Factors. The vector coefficient $\mathrm{W}$ in the weight vector reflects the influence of each index on the object under evaluation [26]. In this paper, the analytic hierarchy process is adopted to determine the weight vector. When the importance of PCI of pavement damage is 1 , it is considered that the importance of rut is $1 / 2$, the importance of flatness is 2 , the importance of antislip is 1 , and the importance of structural strength is 3 . After constructing the judgment matrix $\mathrm{C}$ to find its maximum eigenvalue and eigenvector and normalizing it, the weight coefficient of each index is obtained as $\omega_{1}, \omega_{2}, \omega_{3}$, and $\omega_{4}$ :

$$
C=\left(c_{i j}\right)=\left[\begin{array}{cccc}
c_{11} & c_{12} & \cdots & c_{1 j} \\
c_{21} & c_{22} & \cdots & c_{2 j} \\
\cdots & \cdots & \cdots & \cdots \\
c_{i 1} & c_{i 2} & \cdots & c_{i j}
\end{array}\right]
$$

For the consistency test on matrix C, taking the consistency index,

$$
\mathrm{CI}=\frac{\lambda_{\max }-n}{n-1}
$$

The randomness index RI is shown in Table 2 .

For the consistency test for matrix $\mathrm{C}$ using equation (3), if $\mathrm{CR}<0.1$, then judge matrix $\mathrm{C}$ has good consistency. Otherwise, matrix $\mathrm{C}$ needs to be readjusted:

$$
\mathrm{CR}=\frac{\mathrm{CI}}{\mathrm{RI}}
$$

4.1.5. Result Vector of Fuzzy Comprehensive Evaluation. According to this formula, $b_{i}$ is the i-th membership degree calculated by combining all factors of $W$ and $R$. According to the principle of maximum membership, let $d_{0}=\max \left[b_{i}\right]$, and then consider the i-th comment level in the comment domain $V$ :

$$
\begin{aligned}
D\left[d_{i}\right] & =W \cdot R=\left[\omega_{1}, \omega_{2}, \omega_{3}, \ldots, \omega_{m}\right] \cdot\left[\begin{array}{ccc}
r_{11} & \cdots & r_{1 n} \\
\ldots & \cdots & \cdots \\
r_{m 1} & \cdots & r_{m n}
\end{array}\right] \\
& =\left[b_{1}, b_{2}, \ldots, b_{n}\right] .
\end{aligned}
$$

4.2. Example Analysis. Taking the $\mathrm{K} 23+000-\mathrm{K} 33+000$ upbound line of the Xingtai-Linqing expressway in Hebei Province as an example, the performance evaluation indexes of each item were calculated. The test results are shown in Table 3.

Since PCI $=68.83$, the membership function values at the optimal, good, medium, secondary, and bad levels were calculated, respectively, according to the above calculation formula and obtained after normalization.
TABLE 2: Randomness index RI.

\begin{tabular}{ccccccc}
\hline$n$ & 1 & 2 & 3 & 4 & 5 & 6 \\
\hline RI & 0 & 0 & 0.59 & 0.9 & 1.12 & 1.24 \\
\hline
\end{tabular}

TABLE 3: Section K23-K33 performance evaluation indexes.

\begin{tabular}{lcccc}
\hline Test results & PCI & RQI & RDI & SRI \\
\hline K23-K33 (uplink) & 68.83 & 93.84 & 79.39 & 73.88 \\
\hline & \\
PCI $=68.83, r_{1}=\left[r_{\mathrm{E}}, r_{\mathrm{G}}, r_{\mathrm{M}}, r_{\mathrm{S}}, r_{\mathrm{B}}\right]=\left[r_{11}, r_{12}, r_{13}, r_{14}\right.$, \\
$\left.r_{15}\right]=[0,0.922,1,0.078,0]$ \\
RQI $=93.84, r_{2}=\left[r_{21}, r_{22}, r_{23}, r_{24}, r_{25}\right]=[1,0.411,0,0,0]$ \\
RDI $=79.39, r_{3}=\left[r_{31}, r_{32}, r_{33}, r_{34}, r_{35}\right]=[0.626,1,0.374$, \\
$0,0]$ \\
SRI $=73.88, r_{4}=\left[r_{41}, r_{42}, r_{43}, r_{44}, r_{45}\right]=[0.259,1,0.741$, \\
$0,0]$
\end{tabular}

To sum up, the matrix is obtained $R_{\left(\left[V_{i j}\right]\right)}$ :

$$
R_{\left(\left[v_{i j}\right]\right)}=\left[\begin{array}{ccccc}
0 & 0.922 & 1 & 0.078 & 0 \\
1 & 0.411 & 0 & 0 & 0 \\
0.626 & 1 & 0.374 & 0 & 0 \\
0.259 & 1 & 0.741 & 0 & 0
\end{array}\right] .
$$

Technical indexes of the Xinglin expressway asphalt pavement performance are $\mathrm{C}_{1}$-PCI, $\mathrm{C}_{2}$-RDI, $\mathrm{C}_{3}$-RQI, and $\mathrm{C}_{4}$-SRI. The judgment matrix is constructed by equation (2):

$$
C=\left(c_{i j}\right)=\left[\begin{array}{llll}
c_{11} & c_{12} & \cdots & c_{1 j} \\
c_{21} & c_{22} & \cdots & c_{2 j} \\
\cdots & \cdots & \cdots & \cdots \\
c_{i 1} & c_{i 2} & \cdots & c_{i j}
\end{array}\right] \stackrel{\text { i,jtake4 }}{=}\left[\begin{array}{cccc}
1 & 2 & 1 & 3 \\
1 / 2 & 1 & 1 / 2 & 2 \\
1 & 2 & 1 & 3 \\
1 / 3 & 1 / 2 & 1 / 3 & 1
\end{array}\right] .
$$

For maximum eigenvalues and eigenvectors,

$$
\begin{aligned}
& \sum_{j=1}^{4} c_{1 j}=1 \times 2 \times 1 \times 3=6, \overline{w_{1}}=\left(\sum^{4} c_{1 j}\right)^{1 / 4}=6^{1 / 4}=1.565, \\
& \sum_{j=1}^{4} c_{2 j}=\frac{1}{2} \times 1 \times \frac{1}{2} \times 2=\frac{1}{2}, \overline{w_{2}}=\left(\sum^{4} c_{2 j}\right)^{1 / 4}=\left(\frac{1}{2}\right)^{1 / 4}=0.841, \\
& \sum_{j=1}^{4} c_{3 j}=1 \times 2 \times 1 \times 3=6, \overline{w_{3}}=\left(\sum^{4} c_{3 j}\right)^{1 / 4}=6^{1 / 4}=1.565 \\
& \sum_{j=1}^{4} c_{4 j}=\frac{1}{3} \times \frac{1}{2} \times \frac{1}{3} \times 1=\frac{1}{18}, \overline{w_{4}}=\left(\sum^{4} c_{4 j}\right)^{1 / 4}=\left(\frac{1}{18}\right)^{1 / 4}=0.845 .
\end{aligned}
$$

Normalize the eigenvector $\bar{w}=\left(\overline{w_{1}}, \overline{w_{2}}, \overline{w_{3}}, \overline{w_{4}}\right)^{\mathrm{T}}=$ $(1.565,0.841,1.565,0.485)^{\mathrm{T}}$ :

$$
w_{1}=\frac{\overline{w_{i}}}{\sum_{j=1}^{4} \overline{w_{j}}}, \quad i=1,2,3,4, j=1,2,3,4, \sum_{j=1}^{4} \overline{w_{j}}=4.456 .
$$


TABLE 4: Road performance evaluation results of K23 K33 section.

\begin{tabular}{lccccccc}
\hline Stake & Excellent & Good & Medium & Secondary & Bad & Evaluation & PQI evaluation \\
\hline K23-K24 & 0.555 & 0.723 & 0.444 & 0.157 & 0 & Good & Good \\
K24-K25 & 0.466 & 0.803 & 0.534 & 0.073 & 0 & Good & Good \\
K25-K26 & 0.446 & 0.668 & 0.554 & 0.091 & 0 & Good & Good \\
K26-K27 & 0.535 & 0.823 & 0.464 & 0.058 & 0 & 0 & Good \\
K27-K28 & 0.563 & 0.777 & 0.437 & 0.077 & 0 & Good \\
K28-K29 & 0.646 & 0.879 & 0.488 & 0 & 0 & Medium & Good \\
K29-K30 & 0.259 & 0.596 & 0.670 & 0.318 & 0 & Good & Good \\
K30-K31 & 0.715 & 0.900 & 0.285 & 0.271 & 0 & Good \\
K31-K32 & 0.472 & 0.534 & 0.528 & 0 & 0 & Good \\
K32-K33 & 0.624 & 0.879 & 0.376 & Godium \\
\hline
\end{tabular}

So,

$$
\begin{aligned}
& w_{1}=\frac{1.565}{4.456}=0.351, \\
& w_{2}=\frac{0.841}{4.456}=0.189 \text {, } \\
& w_{3}=\frac{1.565}{4.456}=0.351 \text {, } \\
& w_{4}=\frac{0.485}{4.456}=0.109 \text {, } \\
& \mathrm{CW}=\left[\begin{array}{cccc}
1 & 2 & 1 & 3 \\
1 / 2 & 1 & 1 / 2 & 2 \\
1 & 2 & 1 & 3 \\
1 / 3 & 1 / 2 & 1 / 3 & 1
\end{array}\right]\left[\begin{array}{l}
0.351 \\
0.189 \\
0.351 \\
0.109
\end{array}\right], \\
& \lambda_{\max }=\sum_{i=1}^{n} \frac{(c \cdot w)_{i}}{n \cdot w_{i}}=\frac{1.407}{4 \times 0.351}+\frac{0.758}{4 \times 0.189}+\frac{1.407}{4 \times 0.351} \\
& +\frac{0.437}{4 \times 0.109}=4.01 \text {. }
\end{aligned}
$$

Inspection $\quad \mathrm{CI}=\left(\lambda_{\max }-n\right) /(n-1)=(4.01-4) /(4-$ 1) $=0.0035$ and $\mathrm{CR}=\mathrm{CI} / \mathrm{RI}=0.0035 / 0.90=0.0038<0.1$. It is consistent. Therefore, $w_{1}, w_{2}, w_{3}$, and $w_{4}$ are $0.351,0.351$, 0.189 , and 0.109 , respectively. The weight vector $w=\left(w_{1}, w_{2}, w_{3}, w_{4}\right)^{\mathrm{T}}$, synthesis of the fuzzy comprehensive evaluation result vector by formula (3):

$$
\begin{array}{r}
D=w \cdot R=[0.351,0.351,0.189,0.109] \\
\cdot\left[\begin{array}{ccccc}
0 & 0.922 & 1 & 0.078 & 0 \\
1 & 0.411 & 0 & 0 & 0 \\
0.626 & 1 & 0.374 & 0 & 0 \\
0.259 & 1 & 0.741 & 0 & 0
\end{array}\right] .
\end{array}
$$

The resulting $D=[0.497,0.765,0.502,0.027,0]$. According to the principle of maximum membership, when $i=2, D_{0}=\max \left[d_{i}\right]=0.765$. The corresponding rating is "good."
Similarly, performance evaluation results of uplink can be obtained based on the above model, as shown in Table 4 .

It can be seen from Table 4 that, based on the multiindex comprehensive detection and evaluation adopted by the specification, the performance grade of the old road surface is quantitatively evaluated by using the iterative calculation of fuzzy mathematics, and the evaluation score of each kilometer is more quantitatively intuitive. It highlights the idea of expert evaluation based on traditional detection methods, weakens subjective judgment, highlights objective quantitative evaluation, and breaks through the evaluation mode based on traditional detection methods. The research results provide an innovative theoretical method for the accurate evaluation and analysis of highway pavement performance in semiarid climate regions.

\section{Conclusions}

This paper uses fuzzy mathematics to comprehensively evaluate the pavement performance of Xingtai to Linqing section of Taihang mountains expressway in the semiarid climate zone of Hebei Province. Based on the multi-index comprehensive detection and evaluation adopted by the specification, fuzzy mathematics is used to iteratively calculate and evaluate the performance grade of the old road. The research puts forward the feasibility of the fuzzy mathematics method in highway pavement performance evaluation and obtains the following research conclusions:

(1) This paper statistically analyzes the meteorological and hydrological characteristics of semiarid climate zones, combined with engineering case investigation and analysis. The results show that the types and distribution characteristics of highway pavement diseases are closely related to rainfall, extreme temperature, and temperature difference in semiarid climate areas.

(2) The study uses the factor domain, the comment level domain, the fuzzy relationship matrix, the evaluation factor full vector, and the fuzzy comprehensive evaluation result vector five-step method. The method can be effectively combined with the multiindex comprehensive detection index used in the specification. 
(3) Based on the multi-index comprehensive test and evaluation adopted in the specification, the performance grade of the old road surface was quantitatively evaluated by iterative calculation with fuzzy mathematics, which broke through the evaluation mode based on the traditional detection methods. The research results provide an innovative theoretical method for the accurate evaluation and analysis of highway pavement performance in semiarid climate regions.

\section{Data Availability}

The data used to support the findings of this study are included within the article.

\section{Conflicts of Interest}

The authors declare that there are no conflicts of interest regarding the publication of this paper.

\section{Acknowledgments}

This research was supported by Hebei Provincial Natural Science Foundation Funded Project (E2018201106), Hebei Provincial Department of Education Research Program Key Project (ZD2016073), Hebei Province High-Level Talents Funding Project (B2017005024), and National Natural Science Foundation of China (51808016).

\section{References}

[1] Q. R. Li, Z. Y. Guo, Y. J. Wang, Evaluation of freeway asphalt pavement performance based on PCA-SVM," Journal of Beijing University of Technology: Naturnal Science Eition, vol. 44, no. 2, pp. 283-288, 2018.

[2] J. Zhao, Performance evaluation and prediction model of asphalt pavement, Ph.D. thesis, Dalian University of Technology, Dalian, China, 2008.

[3] (FHWA), U.S. Department of Transportation Federal Highway Administration Office of Management Asset Management Overview, (FHWA), Washington, DC, USA, 2007.

[4] M. Guo and Y. Q. Tan, "Interaction between asphalt and mineral fillers and its correlation to mastics' viscoelasticity," International Journal of Pavement Engineering, 2019.

[5] R. Hass, W. R. Huston, and J. Zaniewshi, Modern Pavement Management, Malabar, Florida: Krieger Publishing Company, Malabar, FL, USA, 1994.

[6] M. Y. Shahin, "Pavement management-PAVER update," in Proceedings of the Annual Meeting of the Transportation Research Board (TRB), National Research Council, Washington, DC, USA, 1999.

[7] K. C. P. Wang, J. Zaniewsk, and G. Way, "Probabilistic behavior of pavements," Journal of Transportation Engineering, vol. 120, no. 3, pp. 358-375, 1994.

[8] B. R. Kulkarni and W. R. Miller, "Pavement Management Systems: Past, Present, and Future," Transportation Research Board, vol. 1853, no. 1, pp. 65-71, 2003.

[9] J. L. Prozzi, Modeling pavement performance by combining field and experimental data, Ph.D. thesis, University of California, Oakland, CA, USA, 2001.
[10] C. Nunoo, Optimization of Pavement Maintenance and Rehabilitation Programming Using Shuffled Complex Evolution Algorithm, Florida International University, University Park, FL, USA, 2001.

[11] W. O. Hadley, SHRP-LTTP Overview: Five-Year Report, SHRP-P-416, Washington, DC, USA, 1994.

[12] D. L. Hao, Evaluation and Analysis of Pavement Performance, Chang'an University, Xi'an, China, 2000.

[13] R. K. Kher and M. I. Darter, Probabilistic Concept and Their Application to AASHO Interim Guide for Design of Rigid Pavements, HRB 466, Washington, DC, USA, 1973.

[14] Pavement Desing and Evalauation Committee, Roads and Transportation Association of Canada, "Output measurement for pavement management studies in canada," in Proceedings of the Third International Conference on the Structural Design of Asphalt Pavements (ICSDAP), University of Michigan, Ann Arbor, MI, USA, 1972.

[15] H. Zhou, K. Xie, and H. M. Hu, "Evaluation and pavement model of expressway to pavement performance," Engineering and Construction, vol. 115, no. 1, pp. 66-69, 2018.

[16] T. Chen, Research on Performance Evaluation Technology and Reinforcement Design Method of Expressway Asphalt Pavement, Chang'an University, Xi'an, China, 2003.

[17] S. Q. Wen, Research on Expressway Pavement Maintenance Decision Based on Combination Prediction and Fuzzy Optimization, Dalian University of Technology, Dalian, China, 2009.

[18] H. M. Li, Research on Maintenance Decision of Asphalt Pavement of Network Expressway Based on Matter-Element Model, Southeast university, Dhaka, Bangladesh, 2017.

[19] J. P. Huang, M. X. Ji, and Y. Z. Liu, "An overview of arid and semi-arid climate change," Progressus Inquisitiones DE Mutatione Climatis, vol. 9, no. 1, pp. 09-14, 2013.

[20] C. Ling, L. Zhou, and F. Gu, "The application of extension theory on pavement performance evaluation," Advanced Materials Research, vol. 168-170, pp. 111-115, 2010.

[21] S. Q. Yang, N. Li, and S. Zhang, "Analysis of design index and structural mechanics of durable asphalt pavement in desert area," Journal of Hebei University: Naturnal Science Edition, vol. 36, no. 6, pp. 574-582, 2016.

[22] S. Q. Yang, W. X. Wu, and N. Li, “Ansys simulation analysis of cement concrete pavement bearing capacity under extra heavy load," Journal of Hebei University: Naturnal Science Edition, vol. 37, no. 6, pp. 561-566, 2017.

[23] S. W. Qi and F. Q. Wu, "Surrounding rockmass quality classification of tunnel cut by TBM with fuzzy mathematics method," Chinese Journal of Rock Mechanics and Engineering, vol. 30, no. 6, pp. 1225-1229, 2011.

[24] J. Bao, Y. Zhang, X. Su, and R. Zheng, "Unpaved road detection based on spatial fuzzy clustering algorithm," EURASIP Journal on Image and Video Processing, vol. 26, no. 12018.

[25] J. X. Tang and X. Y. Li, "On the stability evaluation of the deep subway foundation pit based on the fuzzy mathematical theory," Journal of Safety and Environment, vol. 18, no. 6, pp. 2135-2140, 2018.

[26] X. Li, X. W. Yang, and J. Huang, "Comprehensive evaluation system of cement pavement performance based on fuzzy mathematics," Journal of Lanzhou University: Naturnal Science Edition, vol. 45, no. S1, pp. 88-92, 2009. 


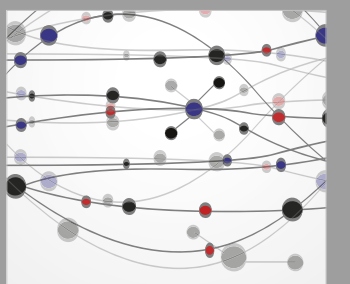

The Scientific World Journal
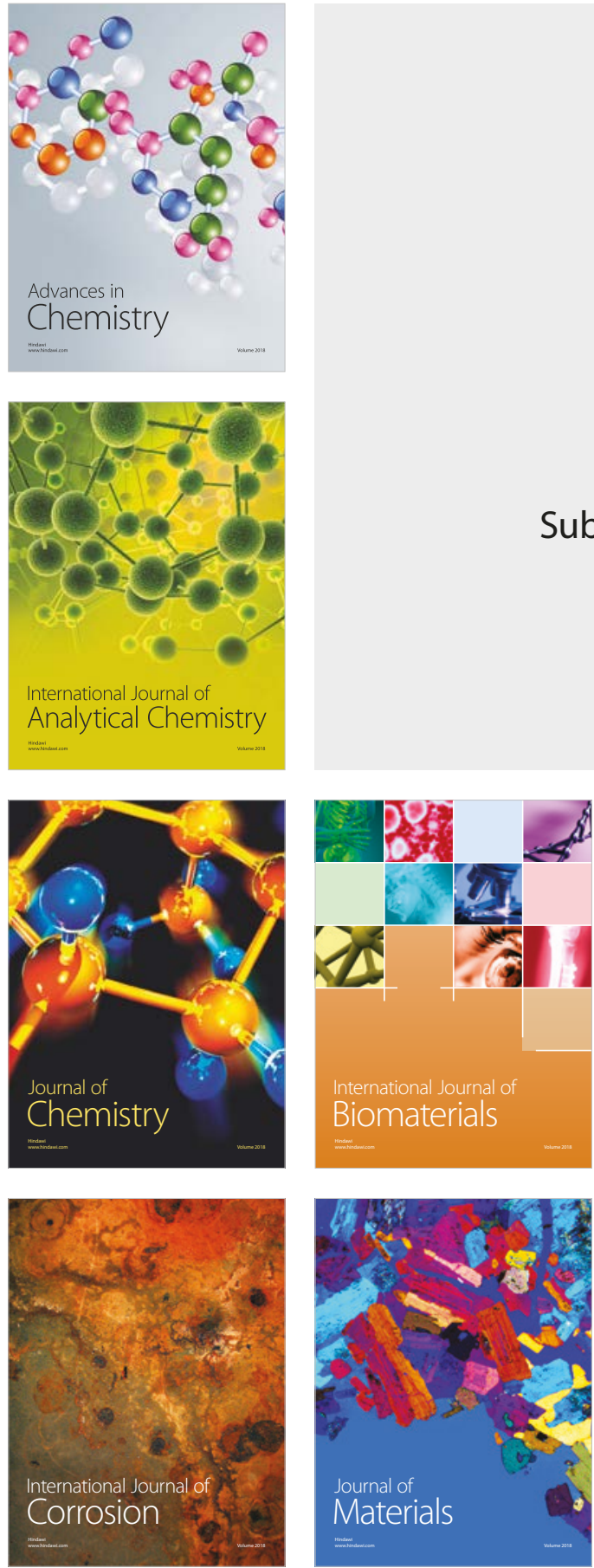

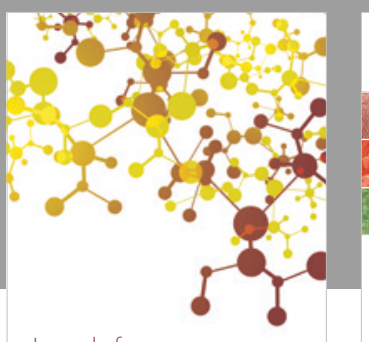

Journal of

Applied Chemistry
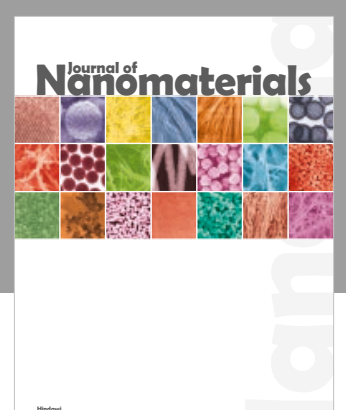

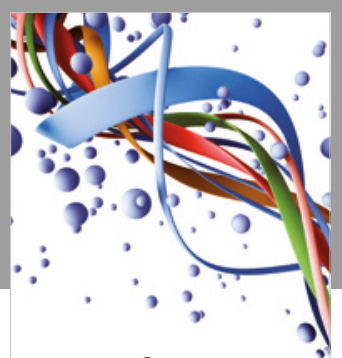

Scientifica

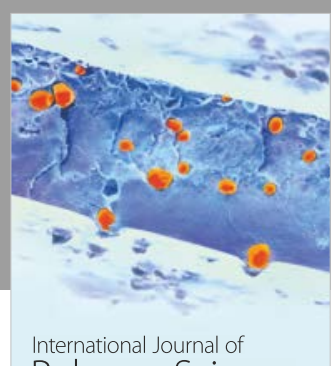

Polymer Science

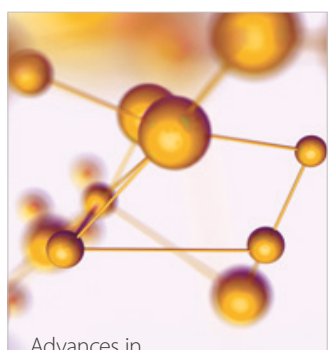

Physical Chemistry
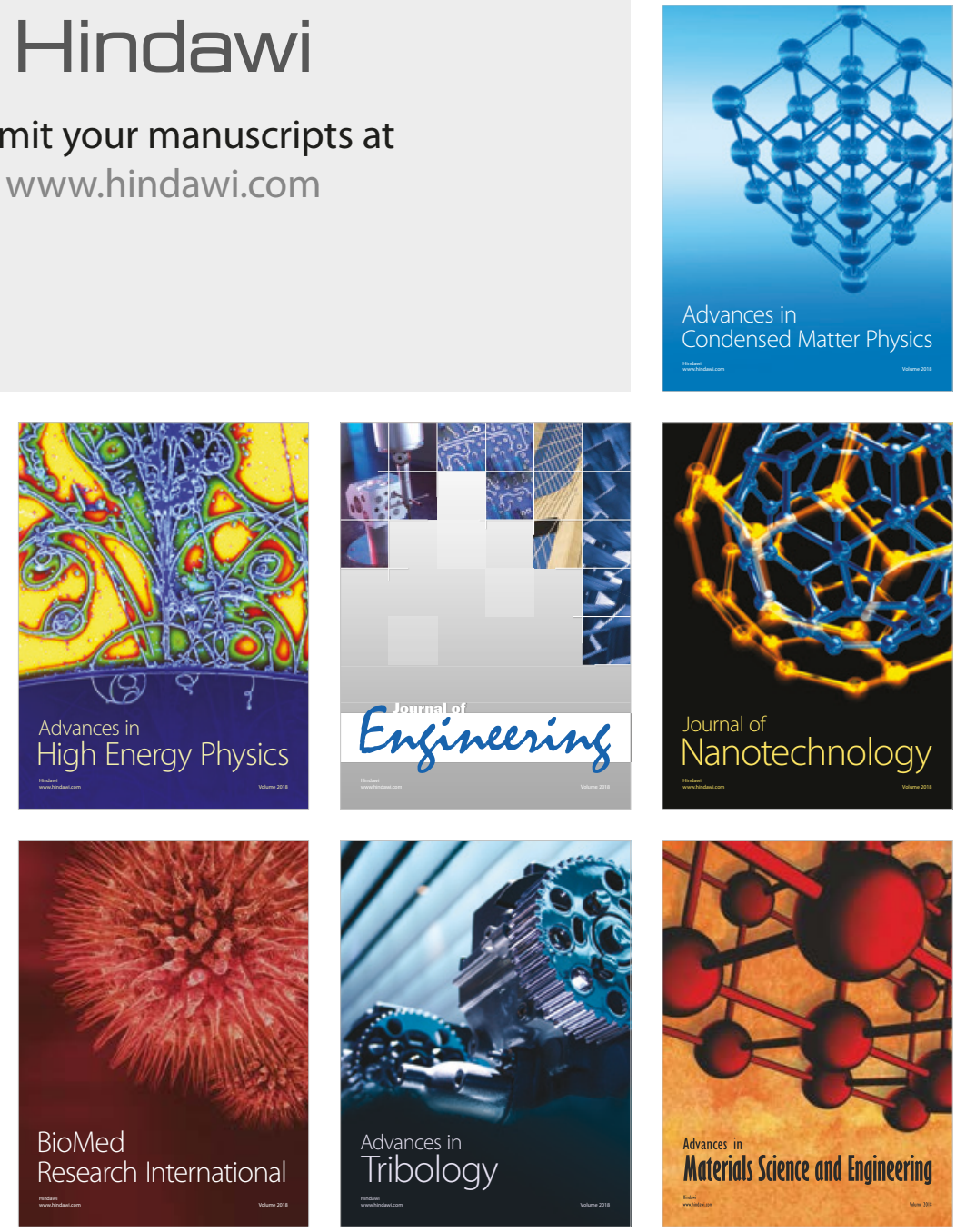